\title{
Self-developed NF- $x$ B inhibitor 270 protects against LPS-induced acute kidney injury and lung injury through improving inflammation
}

\author{
Yanyan $\mathrm{Yu}^{1}$, Xiangqian $\mathrm{Li}^{1}$, Wenpeng $\mathrm{Hu}^{1}$, Shichao Cui ${ }^{2}$, Jiajia Dai ${ }^{1}$, Yanan $\mathrm{Gao}^{1}$, Yiting \\ Zhang ${ }^{1}$, Jiaying Zhu ${ }^{1}$, and Dayong $\mathrm{Shi}^{1}$ \\ ${ }^{1}$ Shandong University \\ ${ }^{2}$ Shanghai Institute of Materia Medica Chinese Academy of Sciences
}

June 22, 2021

\begin{abstract}
Background and Purpose: Sepsis-induced acute kidney injury (AKI) and acute lung injury (ALI) have high morbidity and mortality, with no effective clinically available drugs. Anti-inflammation is effective strategy in the therapy of AKI and ALI. $\mathrm{NF}-x \mathrm{~B}$ is a target for the development of anti-inflammatory agents. The purpose of the study is to evaluate the effect of 270 , self-developed NF- $\varkappa$ B inhibitor, in LPS-induced AKI and ALI. Experimental Approach: LPS-induced macrophages were used to examine the anti-inflammation activity of 270. Sepsis-induced AKI and ALI mice models were established by intraperitoneal injection of LPS $(10 \mathrm{mg} / \mathrm{kg})$ for $24 \mathrm{~h}$. Oral administration 270 for 14 days before LPS stimulation. Plasma, kidney and lung tissues were collected and used for histopathology, biochemical assay, ELISA, RT-PCR, and western blot analyses. Key Results: In vitro, we showed that 270 suppressed the inflammation response in LPS-induced RAW 264.7 macrophages and bone marrow derived macrophages. In vivo, we found that 270 ameliorated LPS-induced AKI and ALI, as evidenced by improving various pathological changes, reducing the expression of pro-inflammation genes, blocking the activation of $\mathrm{NF}-\varkappa \mathrm{B}$ and JNK pathways, attenuating the elevated myeloperoxidase (MPO) activity and malondialdehyde (MDA) content, ameliorating the activated ER stress, reversing the inhibition effect on autophagy in kidney and lung tissues, and alleviating the enhanced plasma level of creatinine (Crea), blood urea nitrogen (BUN) and pro-inflammation cytokines. Conclusions and Implications: Our investigations provides evidence that NF- $x \mathrm{~B}$ inhibitor 270 is a potential drug against LPS-induced AKI and ALI in the future.
\end{abstract}

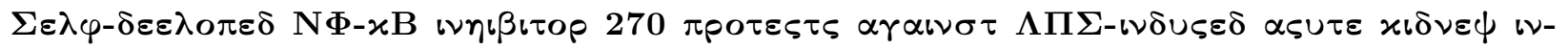

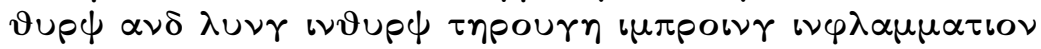

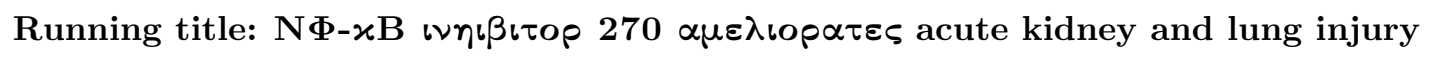

Yan-yan $\mathrm{Yu}^{1}$, Xiang-qian $\mathrm{Li}^{1}$, Wen-peng $\mathrm{Hu}^{1}$, Shi-chao $\mathrm{Cu}^{3}$, Jia-jia Dai ${ }^{1}$, Ya-nan Gao ${ }^{1}$, Yi-ting Zhang ${ }^{1}$, Jia-ying Zhu ${ }^{1}$ and Da-yong $\mathrm{Shi}^{1,2^{*}}$

${ }^{1}$ State Key Laboratory of Microbial Technology, Institute of Microbial Technology, Shandong University, Qingdao, 266200 China

${ }^{2}$ Laboratory for Marine Drugs and Bioproducts of Qingdao National Laboratory for Marine Science and Technology, Qingdao, 266071, China

${ }^{3}$ State Key Laboratory of Drug Research, Shanghai Institute of Materia Medica, Chinese Academy of Science, Shanghai, China

* Correspondence: 
Da-yong Shi, State Key Laboratory of Microbial Technology, Institute of Microbial Technology, Shandong University, Qingdao, 266200 China, E-mail: shidayong@sdu.edu.cn

Word count : 5877 words (excluding Figure legends and References)

Abstract (250 words)

Introduction (632 words)

Methods (1342 words)

Results (2133 words)

Discussion and Conclusions (1216 words)

\section{Acknowledgements}

This work was supported by Certificate of China Postdoctoral Science Foundation Grant (2019M662374), Fundamental Research Funds of Shandong University (2020GN033), National Natural Science Foundation of China (82003787), Shandong Provincial Natural Science Foundation (ZR2020QH364).

\section{Conflict of Interest Statement}

The authors declare no conflicts of interest.

\section{Data Availability Statement}

Data available on request from the authors. The data that support the findings of this study are available from the corresponding author upon reasonable request. Some data may not be made available because of privacy or ethical restrictions.

\section{What is already known}

Anti-inflammation is effective strategy in the therapy of AKI and ALI.

$\mathrm{NF}-\chi \mathrm{B}$ is a target for the development of anti-inflammatory agents

\section{What this study adds}

Compound 270, a self-developed NF- $\chi \mathrm{B}$ inhibitor, possess anti-inflammation activity in vitro.

$\mathrm{NF}-\varkappa \mathrm{B}$ inhibitor 270 has protective effects against LPS-induced septic AKI and ALI in vivo.

\section{What is the clinical significance}

$\mathrm{NF}-x \mathrm{~B}$ inhibitor 270 is a potential candidate medicine in the treatment of AKI and ALI.

$\mathrm{NF}-\varkappa \mathrm{B}$ inhibitor 270 could be used in inflammatory associated diseases.

\section{ABSTRACT}

\section{Background and Purpose:}

Sepsis-induced acute kidney injury (AKI) and acute lung injury (ALI) have high morbidity and mortality, with no effective clinically available drugs. Anti-inflammation is effective strategy in the therapy of AKI and ALI. NF- $\varkappa \mathrm{B}$ is a target for the development of anti-inflammatory agents. The purpose of the study is to evaluate the effect of 270 , self-developed NF- $x$ B inhibitor, in LPS-induced AKI and ALI.

\section{Experimental Approach:}

LPS-induced macrophages were used to examine the anti-inflammation activity of 270 . Sepsis-induced AKI and ALI mice models were established by intraperitoneal injection of LPS $(10 \mathrm{mg} / \mathrm{kg})$ for $24 \mathrm{~h}$. Oral administration 270 for 14 days before LPS stimulation. Plasma, kidney and lung tissues were collected and used for histopathology, biochemical assay, ELISA, RT-PCR, and western blot analyses. 


\section{Key Results:}

In vitro, we showed that 270 suppressed the inflammation response in LPS-induced RAW 264.7 macrophages and bone marrow derived macrophages. In vivo, we found that 270 ameliorated LPS-induced AKI and ALI, as evidenced by improving various pathological changes, reducing the expression of pro-inflammation genes, blocking the activation of NF- $x \mathrm{~B}$ and JNK pathways, attenuating the elevated myeloperoxidase (MPO) activity and malondialdehyde (MDA) content, ameliorating the activated ER stress, reversing the inhibition effect on autophagy in kidney and lung tissues, and alleviating the enhanced plasma level of creatinine (Crea), blood urea nitrogen (BUN) and pro-inflammation cytokines.

\section{Conclusions and Implications:}

Our investigations provides evidence that NF- $\varkappa \mathrm{B}$ inhibitor 270 is a potential drug against LPS-induced AKI and ALI in the future.

\section{Keywords:}

AKI, ALI, inflammation, NF- $x$ B inhibitor 270

\section{Abbreviations}

ALI, acute lung injury; AKI, acute kidney injury; ARDS, acute respiratory distress syndrome (ARDS); BMDMs, bone marrow derived macrophages; BUN, blood urea nitrogen; CETSA, cellular thermal shift assay; CKD, chronic kidney disease; CMC-Na, sodium carboxymethylcellulose; COX-2, cyclooxygenase-2; Crea, creatinine; ER, endoplasmic reticulum; ESKD, end-stage kidney disease; GAPDH, glyceraldehydes3-phosphate dehydrogenase; HE, haematoxylin and eosin; HG-DMEM, high-glucose Dulbecco's modified eagle's medium; IL-, interleukin; I $\chi$ B- $\alpha$, B-cells inhibitor alpha; IKK, I $\chi$ B kinase; JNK, Jun-aminoterminal kinase; KIM1, kidney injury molecule 1; LC3A, Light Chain 3 A; LPS, lipopolysaccharide; MCP 1, monocyte chemoattractant protein; MDA, malondialdehyde; mM-CSF, mouse macrophage colony stimulating factor; MPO, myeloperoxidase; NF- $\nsim$ B, nuclear factor-kappa B; NGAL, neutrophil gelatinase-associated lipocalin; Nos2, inducible NO synthase; p62, Sequestosome 1; PTL, parthenolide; TNF- $\alpha$, tumour necrosis factor $\alpha$. Veh, the control group.

\section{INTRODUCTION}

Acute kidney injury (AKI) is featured by a sharp decline and even loss of kidney function, and the eventual development of chronic kidney disease (CKD) and end-stage kidney disease (ESKD) (Ryter et al. , 2015; Pak et al. , 2020). AKI is not a mere singular organ injury, there is increasing evidence that acute kidney injury directly contributes to remote injury in the lung, heart, liver, immunologic, and other organ systems (Gumbert et al. , 2020). Acute lung injury (ALI) is characterized by rapid alveolar injury and respiratory disorder, which may develop into its most severe form acute respiratory distress syndrome (ARDS) (Nie et al. , 2019). Both AKI and ALI are diseases with high morbidity and mortality and high healthcare costs (Badamjav et al. , 2020; Pak et al. , 2020). Despite extensive efforts and substantial large-scale clinical trials have been made to develop effective therapeutic strategies for AKI and ALI. There are still no approved methods or agents to protect against AKI and ALI are available at present, which highlights the urgent require for the identifying novel pharmacological drugs for AKI and ALI (Zeng et al. , 2017; Jiang et al. , 2019).

Epidemiological analysis has shown that sepsis is common pathology of AKI and ALI patients (Silveira et al. , 2021). Sepsis is manifested by systemic inflammation, leading to multiple organs dysfunction, and the kidneys and lungs are the most vulnerable organs (Ibrahim et al. , 2020). Lipopolysaccharide (LPS) also known as toxic constituent of Gram-negative bacterial, is a crucial pathogenic factor of sepsis. LPS has widely been applied to mimic sepsis-related AKI and ALI in animal models via activating cascade inflammatory responses and synthesis of enormous cytokines, which exhibit similar pathological changes to those found in human infectious sepsis (Juet al. , 2018; Islam et al. , 2019). Inflammation is usually recognized as a defensive response against various invasion, however excessive inflammation always cause extensive tissue or 
organ injury and even systemic dysfunction (Nie et al. ,2019). Inflammatory cells infiltration and generation of pro-inflammatory cytokines play a pivotal role in the pathogenesis of AKI and ALI (Islam et al. , 2019; Nie et al. , 2019). Strategies targeting the exaggerated inflammatory response resulted in reduction of the damage severity of AKI and ALI. Anti-inflammatory drugs may be an effective option for the prevention of LPS-induced septic AKI and ALI (Chen et al. , 2019; Silveira et al. , 2021).

The nuclear factor-kappa $\mathrm{B}(\mathrm{NF}-\varkappa \mathrm{B})$ is a crucial transcriptional factor controlling genes that encode for various pro-inflammatory cytokines, such as tumour necrosis factor $\alpha$ (TNF- $\alpha$ ), interleukin-6 (IL-6) and interleukin-1 $\beta$ (IL-1 $\beta$ ) and monocyte chemoattractant protein (MCP) 1 , which is a critical target for the development of anti-inflammatory agents. Numerous compounds have been screened for anti-inflammatory activities by suppressing NF- $x \mathrm{~B}$ (Huanget al. , 2019). NF- $\chi \mathrm{B}$ exists in an inactive form in the cytoplasm bound to the B-cells inhibitor alpha $(\mathrm{I} \varkappa \mathrm{B}-\alpha)$. Upon a series of activation after LPS stimulation, I $x \mathrm{~B}$ kinase (IKK) would be activated, leading to I $\chi \mathrm{B}-\alpha$ activation and degradation, and the dissociated NF- $\chi \mathrm{B}$ could translocate into the nucleus and promote the transcription of inflammatory mediators (Zhang et al. , 2018b). Accumulating evidence suggests that NF- $\chi \mathrm{B}$ signaling pathway plays a significant role in LPS-induced septic AKI and ALI mice, and NF- $\varkappa \mathrm{B}$ is an attractive therapeutic target for LPS-induced septic AKI and ALI (Huang et al. , 2019; Ibrahim et al. , 2020).

In our study, a series of NF- $\chi \mathrm{B}$ inhibitors were designed and synthesized, and we screened their antiinflammatory activity using an NF- $\varkappa \mathrm{B}$ reporter assay. We found 270 exhibited better inhibitory effect, and suppressed the LPS-induced inflammation response in vitro through disturbing NF- $\chi \mathrm{B}$ and JNK signaling pathway. Furthermore, we evaluated the protective abilities of 270 on LPS-induced septic AKI and ALI mice and explored its underlying molecular mechanisms in vivo. The purpose of this study is to provide a novel anti-inflammation drug for the treatment of AKI and ALI.

\section{METHODS}

\subsection{Materials}

Antibodies against I $\chi \mathrm{B}$ kinase $\beta$ (IKK, \#8943), phospho-IKK $\alpha / \beta$ (Ser176/180) (p-IKK, \#2697), B-cells inhibitor alpha (I $\chi \mathrm{B}-\alpha, \# 4814)$, nuclear factor (NF- $\chi \mathrm{B})$ (p65, \#4764), phospho-NF- $\chi \mathrm{B}$ p65(Ser536) (NF$\varkappa \mathrm{B}, \# 3033)$, The stress-activated protein kinase/Jun-aminoterminal kinase SAPK/JNK (JNK, \#9252), phosphor-SAPK/JNK (Thr183/Tyr185) (p-JNK,\#9251), BiP (\#3309), CHOP (\#2895), Sequestosome 1 (p62, \#39749), Light Chain 3 A (LC3A, \#4599), and glyceraldehydes-3-phosphate dehydrogenase (GAPDH, \#5174) were purchased from Cell Signaling Technology; ELISA kits used in measurement of plasma parameters are as follows: Mouse MCP 1 (Boster, EK0568), Mouse IL-6 (Boster, EK0411), Mouse IL-1 $\beta$ (Boster, EK0394), Mouse TNF- $\alpha$ (Boster, EK0527). Lipofectamine 3000 reagent was obtained from Invitrogen. Parthenolide (PTL) was purchased from MedChem Express (MCE). Mouse macrophage colony stimulating factor (mM-CSF). Tumor necrosis factor (TNF)- $\alpha$ lipopolysaccharide (LPS) were obtained from Sigma Aldrich. Luciferase substrates were purchased from Vazyme. High-glucose Dulbecco's modified eagle's medium (HG-DMEM) was purchased from Hyclon.

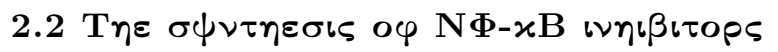

The classic synthesis method of chalcone derivatives is to condense substituted acetophenone and substituted benzaldehyde in alkaline alcohol solution. Because the phenolic hydroxyl group is easy to be oxidized, especially under alkaline conditions. The reaction yield of hydroxychalcone is very low in the classical synthesis of hydroxychalcone. So we explored a method of preparing hydroxychalcone by direct condensation reaction under the catalysis of thionyl chloride. Using thionyl chloride as a catalyst and ethanol as a solvent, hydroxyacetophenone and hydroxybenzaldehyde are condensed at room temperature for 24 hours to obtain various hydroxychalcone derivatives. The detail information is showed in the supplementary information.

\subsection{Cell transfection and luciferase assay}

HEK-293T cells cultured in 96-well plate were transiently transfected with NF- $\varkappa$ B-responsive luciferase reporter plasmid using Lipofectamine 3000 transfection reagent. The cells were cultured for $24 \mathrm{~h}$ and treated 
with the compounds together with TNF- $\alpha(100 \mathrm{ng} / \mathrm{ml})$ for $6 \mathrm{~h}$. Luciferase activity was determined using luciferase assay kits according to the manufacturer's instructions.

\subsection{Cellular thermal shift assay (CETSA)}

HEK-293T cells transfected with NF- $x$ B plasmid and RAW 264.7 macrophages were incubated with DMSO or 270 for $3 \mathrm{~h}$. Cells were harvested and resuspended in PBS and divided into four aliquots. Then cells were heated at different temperatures by $\mathrm{T} 100^{\mathrm{TM}}$ Thermal Cycler (Bio-Rad). The heated cells were freezethawed three times with liquid nitrogen and followed by centrifugation at $20,000 \times \mathrm{g}$ for $20 \mathrm{~min}$ at $4{ }^{\circ} \mathrm{C}$. The supernatants were harvested and loading buffer was added before boiling. Protein levels were measured by western blotting.

\subsection{RAW 264.7 cells culture and treatment}

RAW 264.7 cells were purchased from Cell Bank, and cultured in HG-DMEM medium containing 10\% FBS. All cells were seeded into 24 -well cell culture plates at a concentration of approximately $3 \times 10^{5}$ cells per well, maintained in a humidified atmosphere of $5 \% \mathrm{CO}_{2}$ at $37 @ \mathrm{C}$, and allowed to grow for $24 \mathrm{~h}$. LPS was used to trigger inflammatory responses. RAW 264.7 macrophages were treated with LPS alone $(100 \mathrm{ng} / \mathrm{mL})$ or LPS with 270 for $24 \mathrm{~h}$, harvested cells were used for RNA extraction. RAW 264.7 cells were pretreated with 270 for $4 \mathrm{~h}$ before LPS (100 ng/mL) treatment for $30 \mathrm{~min}$, and cells lysates were obtained for western blot.

\subsection{Bone marrow derived macrophages isolation, culture and treatment}

Bone marrow derived macrophages (BMDMs) were prepared as described (Zhao et al. , 2018). Briefly, BMDMs were isolated from the male C57BL/6J mice at the age of 9 or 10 weeks, and cultured in HGDMEM supplemented with 10\% FBS and mM-CSF $(20 \mathrm{ng} / \mathrm{mL})$, and the medium was replaced on the 4th day. Cells were maintained for 7 days to differentiate into mature macrophages. The mature BMDMs were treated with LPS alone $(100 \mathrm{ng} / \mathrm{mL}$ ) or along with 270 for $24 \mathrm{~h}$, the medium was used for ELISA test, and cells were used for RNA extraction. The mature BMDMs were pretreated with 270 for $4 \mathrm{~h}$ before LPS (100 $\mathrm{ng} / \mathrm{mL}$ ) stimulation for $30 \mathrm{~min}$, and cells lysates were obtained for western blot. In the process, mM-CSF $(20 \mathrm{ng} / \mathrm{mL}$ ) needed to be added to the HG-DMEM containing $10 \%$ FBS to maintain the mature state of BMDMs.

\subsection{RNA isolation and quantitative RT-PCR}

Total RNA was isolated from cells and tissues $(20 \mathrm{mg}$ ) using TRIzol reagent (Invitrogen). The cDNA was prepared using PrimeScript reverse transcriptase (TaKaRa) according to the manufacturer's instructions. After a 10-fold dilution, the cDNAs were amplified using $2 \times$ SYBR Green qPCR master mix (Biotool) and a Stratagene Mx3005P (Agilent Technologies). The primers sequences were used in the paper showed in the supplementary information Table 1.

\subsection{Protein isolation and Western blot}

Proteins were extracted from cells and tissues $(30 \mathrm{mg})$ with radioimmunoprecipitation assay (RIPA) buffer, denatured with loading buffer, resolved with $10 \%$ SDS-PAGE gels, electroblotted onto NC membranes, and then blocked with $5 \%$ nonfat milk. The membranes were incubated with different primary antibodies, followed by incubation with the relative secondary antibodies, and the blots were analyzed by ImageJ.

\subsection{Animal model}

All animal experiments were approved by the Animal Care and Use Committee of Shandong University, where the experiments were conducted. All animals were housed in a temperature-controlled room $\left(22 \pm 2{ }^{\circ} \mathrm{C}\right)$ with a light/dark cycle of $12 \mathrm{~h}$. Male C57BL/6J mice at the age of 8 weeks were randomly divided into four groups (n $=8$ ): the control group (Veh), which received an equal amount of $0.5 \%$ sodium carboxymethylcellulose (CMC$\mathrm{Na})$ solution; LPS-treated group (10 mg/kg LPS), which received LPS (10 mg/kg) only via intraperitoneal injection; 270-treated groups (25 mg/ $/ \mathrm{kg}$ and $50 \mathrm{mg} / \mathrm{kg}$, LPS-270-(25) and LPS-270-(80)), which received 270 at different doses $(25,50 \mathrm{mg} / \mathrm{kg})$ via intragastric administration before LPS stimulation. All mice were 
consecutively given 270 or CMC-Na for 14 days, at the last day, all mice (except for those in the control group) were treated by intraperitoneal injection with $10 \mathrm{mg} / \mathrm{kg}$ body weight LPS to induce septic acute kidney and lung injury. After $24 \mathrm{~h}$ of LPS stimulation, all experimental mice were sacrificed; fasting blood was collected and used for cytokine determination, partial kidney and lung tissues were fixed and used for haematoxylin and eosin (HE) staining, remaining tissues were carefully harvested, immediately frozen in liquid $\mathrm{N}_{2}$, and stored at $-80 \operatorname{deg} \mathrm{C}$ for subsequent analysis.

\subsection{Biochemical tests and detection of cytokines}

The concentrations of TNF- $\alpha$, IL-1 $\beta$, IL- 6 and MCP 1 in mice plasma and BMDMs supernatants were detected using ELISA kits, according to the manufacturer's instructions. To evaluate kidney function, urea nitrogen (BUN) and creatinine (Crea) levels in mice plasma were estimated using commercially available kits (Xinjiankangcheng), and performed following the manufacturer's protocol.

\subsection{Histopathological Evaluation}

Mice were sacrificed $24 \mathrm{~h}$ after LPS challenge. Kidney and lung tissues were fixed with $4 \%$ paraformaldehyde $48 \mathrm{~h}$. The tissues were embedded in paraffin wax and then cut into $4-\mu \mathrm{m}$-thick sections for HE staining. The results of HE staining were observed with a light microscope.

\subsection{Determination of MPO and MDA}

About $30 \mathrm{mg}$ of left upper kidney and lung tissues were homogenated in cold saline solution at a ratio of 1:10 (weight:volume). Myeloperoxidase (MPO) activity is a biochemical marker for the infiltration of neutrophils and macrophages. Malondialdehyde (MDA) is an indicator of oxidant stress (Jiang et al. , 2019). MPO activity and MDA content were measured using a commercially available kit following manufacturer's instructions (Nanjing Jiancheng Bioengineering Institute). Data were calculated as the absorbance after normalization by the protein content.

\subsection{Statistical analysis}

The data and statistical analysis comply with the recommendations of the British Journal of Pharmacology on experimental design and analysis in pharmacology. Animals were randomly allocated to each experimental group, and histological analysis was performed in a blinded manner. All other experiments were not performed in a blinded manner because the measurements could not be affected by personal bias. Data are presented as mean \pm SEM. and were analysed using Student's t-test or one-way ANOVA followed by Bonferroni's for multiple comparison test. Statistical analyses involved using GraphPad Prism 7.0 (RRID: SCR_002798). The criterion for statistical significance was $\mathrm{P}<0.05$. Statistical analysis was undertaken only for studies in which each group size was at least $n=5$. The declared group size is the number of independent values, and data analysis was done using these independent values.

\section{RESULTS}

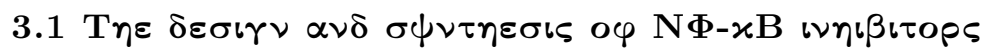

Bromophenol derivatives are unique compounds that exist in many marine organisms, such as algae, ascidians and sponges (Utkina et al. , 2001). The structural specificity of bromophenols makes them exhibit a variety of biological activities, including enzyme inhibition (Lee et al. , 2007), anti-oxidation (Fu et al. , 1995), anti-inflammatory (Wiemer et al. , 1991), anti-microbial (Xu et al. , 2003) and cytotoxic activities (Xuet al. , 2004), etc., which shows a good prospect for the development of new drugs. NF- $\chi \mathrm{B}$ is a significant target for development anti-inflammation drugs, hence, we designed and synthesized various NF- $x \mathrm{~B}$ inhibitors (Figure 1 ), and we screened their transcriptional inhibitory activity using NF- $\chi \mathrm{B}$ reporter assay. The activity studies of compounds 238-242 found that the hydroxyl of $\mathrm{R}_{1}$ and the bromine of $\mathrm{R}_{2}$ are important for inhibitory activity, such as compounds 239 and 241. The activity studies of compounds 242-268 found that hydroxyl or methoxyl of $\mathrm{R}_{6}$ are important for inhibitory activity, such as compounds 259,265 and 268 . The activity studies of compounds 269-280 found that hydroxyl or methoxyl of $\mathrm{R}_{5}$ give compounds 269 and 270 (Figure $2 \mathrm{~A})$ similar high inhibitory activity at $10 \mu \mathrm{M}$ (S Table2). We further evaluated in NF- $\chi \mathrm{B}$ luciferase assay at 
different dosages. Our results showed that 269 and 270 exhibited inhibition with $\mathrm{IC}_{50}$ values $5.91 \mu \mathrm{M}$ and $2.49 \mu \mathrm{M}$, and PTL as a positive control (Figure 2B), suggesting that compound 270 with the methoxy group at the $\mathrm{R}_{5}$ position and the hydroxyl group at the $\mathrm{R}_{6}$ position showed the highest $\mathrm{NF}-\varkappa \mathrm{B}$ transcriptional inhibitory activity, and 270 may be an NF- $x \mathrm{~B}$ inhibitor.

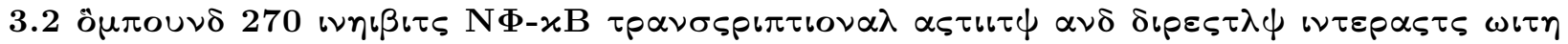
$\mathrm{N} \Phi-\varkappa \mathrm{B}$ ๙ $\mathrm{HEK} 293 \alpha \nu \delta \mathrm{PA} \Omega 264.7 \varsigma \varepsilon \lambda \lambda \varsigma$

CETSA method directly detects the interaction between the drug molecule and target protein in cells, as the binding of molecule can increase the thermal stability of protein (Martinez Molina et al. , 2013).Thus we investigated the binding ability of 270 to NF- $\varkappa$ B in HEK 293 cells (transfected with an NF-kB expression plasmid) and RAW 264.7 macrophages. Cells were incubated separately with DMSO and 270 for $3 \mathrm{~h}$. Then cells were heated at the indicated temperatures and harvested cells were analyzed by western blotting. Our results indicated that 270 treatment protected NF- $\chi \mathrm{B}$ protein from destabilization in HEK 293 cells (transfected with an NF-kB expression plasmid) (Figure 2C,D) and RAW 264.7 macrophages (Figure 2E,F) compared to DMSO, suggesting that 270 directly interacts with $\mathrm{NF}-\varkappa \mathrm{B}$ proteins.

\subsection{Compound 270 suppresses the LPS-induced inflammatory response in RAW 264.7 macrophages}

Exposure to LPS can trigger inflammatory cells (ie, macrophages and neutrophils) infiltration, contributing to excessive inflammatory reaction via NF- $\chi \mathrm{B}$ and JNK signaling pathways (Chen et al. , 2019). Macrophages are the principal immune cells of biosynthesis of pro-inflammatory cytokines, which act as the first line of defense in the kidney and lung, and exert a vital function in the molecular mechanisms of LPS-induced septic AKI and ALI (Huanget al. , 2019). First, we examined the effect of 270 on LPS-induced inflammatory response in RAW 264.7 macrophages. The activation of NF- $x \mathrm{~B}$ occurs through phosphorylation of IKK, leading to the degradation of $\mathrm{I} \chi \mathrm{B}-\alpha$ (Zhang et al. , 2018a). In addition, JNK can directly activate NF- $x \mathrm{~B}$ by promoting $\mathrm{I} \chi \mathrm{B}-\alpha$ degradation, $\mathrm{NF}-\chi \mathrm{B}$ and JNK can coordinate the LPS-induced inflammatory response (Grynberg et al. , 2017). Thus we supposed that 270 may interfere with the NF- $x \mathrm{~B}$ and JNK signaling pathway proteins. So we examined the effect of 270 on the levels of I $\chi \mathrm{B}-\alpha$, and phosphorylation of IKK, NF- $x$ B and JNK in LPS-induced RAW 264.7 macrophages via western blotting. Our results proved that 270 decreased LPS-dependent IKK, NF- $\varkappa$ B and JNK phosphorylation, alleviated LPS-induced I $\chi \mathrm{B}-\alpha$ degradation in dose-dependently (Figure 3A,B). Upon activation by LPS, NF- $\chi \mathrm{B}$ translocate to the nucleus to promote transcription of inflammation genes. Thus we hypothesized that 270 may down-regulate the expression of pro-inflammation genes by disturbing with $\mathrm{NF}-x \mathrm{~B}$ and JNK signaling pathways. Next, we investigated the expression of inflammatory genes by RT-PCR in LPS-induced RAW 264.7 macrophages in the presence or absence of 270. Our data indicated that the high mRNA levels of TNF- $\alpha$, IL-1 $\beta$, IL-6, MCP 1, Nos2 and COX2 caused by LPS were reduced in 270 treated RAW 264.7 cells (Figure 3C), advising that 270 could attenuate LPS-induced inflammation response.

3.4 Compound 270 ameliorates the LPS-induced inflammatory response in bone marrow derived macrophages (BMDMs)

To further clarify that whether 270 possess the direct inhibitory effect on inflammation in macrophages, we treated primary mouse BMDMs with LPS alone or combined with 270 and then detected the NF- $x \mathrm{~B}$ and JNK signaling pathways, the secretion of inflammatory cytokines and the expression of pro-inflammatory genes. We found that the LPS-induced activation of NF- $x \mathrm{~B}$ and JNK signaling pathways in BMDMs were blocked by the pretreatment of 270 , evidenced by the reduction phosphorylation of IKK, NF- $\varkappa \mathrm{B}$ and JNK, and prevention degradation of $\mathrm{I} \alpha \mathrm{B}-\alpha$ in a dose-dependent manner (Figure $4 \mathrm{~A}, \mathrm{~B}$ ). The release of TNF- $\alpha$, IL-6 and MCP 1 from the BMDMs triggered by LPS was also mitigated by 270 administration (Figure $4 \mathrm{C})$. Moreover, 270 remarkably down-regulated the over-expression of IL-1 $\beta$, IL-6, MCP 1, Nos2 and COX2 in LPS-stimulated BMDMs (Figure 4D). In line with the inhibitory ability in RAW 264.7 macrophages. Intriguingly, the repressive effects of 270 on the LPS-induced inflammatory response were more dramatic in BMDMs than in RAW 264.7 macrophages. Our investigations revealed that NF- $\varkappa$ B inhibitor 270 can against 
macrophage-mediated inflammatory response, which may have potential therapeutic effect in inflammationrelated diseases.

3.5 Compound 270 alleviates the excessive biosynthesis of inflammatory cytokines induced by intraperitoneal injection of LPS in C57BL/6J mice

Our observations had demonstrated that the anti-inflammation activity of 270 on LPS- induced macrophages in vitro. Subsequently, we further evaluated the plausible effect of 270 in vivo. LPS, the main pathogenic factor of sepsis, activates a cascade of inflammatory response, which could result in damage to organs. LPS is the pivotal stimulus for the massive production of various inflammatory mediators, which has widely been applied to establish sepsis-associated AKI and ALI mice models (Ju et al. , 2018; Islam et al. , 2019). So we determined the effect of oral administration with 270 on mice injected intraperitoneally with LPS (10 $\mathrm{mg} / \mathrm{kg}$ ) for $24 \mathrm{~h}$. The effect of pretreatment with 270 on food take and body weight was firstly determined, there were no obvious differences among the various groups (Figure 5A). Whereas, the kidney index of mice in the LPS group was significantly higher than that in the Veh group, and pretreatment with 270 markedly relieved the kidney index of LPS-induced mice, and no distinct changes were discovered in the liver and lung index among the various groups (Figure 5B). The plasma levels of TNF- $\alpha$, IL-1 $\beta$, IL-6 and MCP 1 were dramatically elevated after intraperitoneal injection of LPS, and these alterations of pro-inflammatory cytokines following LPS challenge were remarkably ameliorated by administration with 270 (Figure 5C). Our findings reflected that 270 have anti-inflammation activity in vivo and may exert direct effect on kidney in LPS-induced mice.

3.6 Compound 270 improves the mice acute kidney injury caused by intraperitoneal injection of LPS through attenuating renal inflammation state

The kidney is one of the most vulnerable organs during LPS stimulated sepsis (Zhao et al. , 2020). Histological detection was carried out to assess the degree of injury of renal in LPS injection mice. Analysis of the pathological sections of mice renal in Veh group displayed intact tubular and glomerular structure, a visible nucleus, the cytoplasm was uniformly stained, no degeneration occurred, and no apparent inflammation. In contrast, the LPS-injected mice suffered from severe renal pathological damage, characterized by edema of renal tubular epithelial cells, tubular dilatation, brush border disappearance, tubular cell vacuolization, cytoplasmic degeneration, cells exfoliation, intratubular casts formation and inflammatory cells infiltration. Strikingly, the substantial pathological abnormal was notably mitigated in LPS-induced mice after pretreatment with 270 (Figure 6A).

Creatinine (Crea) and blood urea nitrogen (BUN) are important indicators of the severity of renal dysfunction (Zhaoet al. , 2020). In agreement with the histological renal morphology, compare with Veh group mice, plasma Crea and BUN levels were significantly increased in LPS-treated mice, and administration with 270 obviously restored LPS-triggered changes in a dose-dependent manner (Figure 6B). Neutrophil gelatinaseassociated lipocalin (NGAL) and kidney injury molecule 1 (KIM1) are specific tubular injury biomarkers (Jiang et al. , 2019). As expected, the transcription levels of NGAL and KIM1 were dramatically enhanced in the kidneys after LPS exposure for up to $24 \mathrm{~h}$, and pretreatment with 270 remarkably reversed renal anomalous mRNA expression of NGAL and KIM1 caused by the intraperitoneal administration of LPS (Figure 6C).

In order to examine whether 270 can improve mice kidney tissue inflammation state after LPS injection, we evaluated the expression of inflammatory genes and the activity of NF- $x \mathrm{~B}$ and JNK signaling pathways. The mRNA levels of TNF- $\alpha$, IL-1 $\beta$, IL-6, MCP 1 , Nos2 and COX2 in kidney tissue were observably elevated in response to LPS, which were signally prevented by pretreatment with 270 (Figure 6D). Furthermore, LPS markedly upregulated the phosphorylation of IKK, NF- $\varkappa$ B and JNK, and increased the degradation of I $\varkappa \mathrm{B}-\alpha$ in renal tissue, and all these alterations were abrogated by administration with 270 in a dose-dependent manner (Figure 6E,F). Collectively, our investigations concluded that 270 could protect from acute AKI challenged with LPS.

3.7 Compound 270 relieves the mice acute lung injury triggered by intraperitoneal injection 


\section{of LPS through eliminating lung inflammation state}

Despite oral administration with 270 does not affect the lung index of mice injected LPS, but 270 reduced the plasma content of pro-inflammation cytokines. Uncontrolled inflammation is an indispensable mechanism of ALI, and TNF- $\alpha$, IL-1 $\beta$ and IL-6 are critical mediators involved in the development of LPS-induced septic ALI (Huang et al. , 2019). So we assessed the effect of 270 on ALI. To detect the histological variations, lung tissues underwent HE staining. There were no visible histopathological lesions in the Veh group, and lung tissues from the LPS group presented significant thickening of the alveolar septa, the collapse of the alveolar and interstitial infiltration of inflammatory cells, while 270 pretreatment effectively reduced these serious pathological variations attributed to LPS (Figure 7A). The mRNA levels of associated genes in lung determined by RT-PCR, and the expression of TNF- $\alpha$, IL-1 $\beta$, MCP 1 and Nos2 in LPS-challenged mice lung tissues was evidently enhanced, meanwhile the administration of 270 dose dependently eliminated the increase of these pro-inflammation genes (Figure 7B). Next, we tested the effect of 270 on the key proteins related to NF- $x \mathrm{~B}$ and JNK signaling pathways. Compared with the Veh group, the protein levels of p-IKK, $\mathrm{p}-\mathrm{NF}-\varkappa \mathrm{B}$ in LPS group lung tissues were up-regulated, and the protein level of $\mathrm{I} \varkappa \mathrm{B}-\alpha$ was down-regulated. Compared with the LPS group, the expression of p-IKK, p-NF- $\chi \mathrm{B}$ and p-JNK protein was reduced by 270 , and the level of $\mathrm{I} \chi \mathrm{B}-\alpha$ protein was elevated after treatment with 270 (Figure $7 \mathrm{C}, \mathrm{D}$ ). Our observations implied that 270 can protect mice against LPS-induced septic ALI.

\subsection{Compound 270 affects various molecular mechanisms involved in the pathogenesis of acute kidney and lung injury challenged with intraperitoneal injection of LPS}

NF- $\chi \mathrm{B}$ signaling pathways activated by LPS play vital roles in inflammation, oxidative stress, endoplasmic reticulum (ER) stress and autophagy, all these participate in the development of LPS-induced acute kidney and lung injury (Muriach et al. , 2014). Myeloperoxidase (MPO) is a specific indicator of neutrophils and macrophages infiltration and indirectly reflects the severity of inflammation in injury tissues (Huang et al. , 2016). Our results pointed that MPO activity of kidney and lung tissues was higher in the LPS-treated group compared with the Veh group, and pretreatment with 270 dose dependently reduced mice renal (Figure 8A) and lung (Figure 8E) MPO activity compared with the LPS group.

Malondialdehyde (MDA) is considered to be a marker reflecting the level of oxidative stress, and its formation could aggravate damage to cell membranes (Niu et al. , 2019). The MDA content of mice in kidney and lung tissues was significantly increased after $24 \mathrm{~h}$ LPS injection, while 270 pretreatment inhibited the enhancement of MDA content in renal (Figure 8B) and lung (Figure 8F) tissues induced by LPS. To further elucidate the protective role of 270 in LPS-induced AKI and ALI, the protein levels of ER stress markers (BiP and CHOP) and autophagy-related factors (LC3A and p62) were measured via western blotting. Our results showed that LPS obviously promoted the expression of $\mathrm{BiP}$ and $\mathrm{CHOP}$ proteins in mice kidney and lung tissues, and the up-regulation of the $\mathrm{BiP}$ and $\mathrm{CHOP}$ proteins in kidney (Figure 8C,D) and lung (Figure 8G,H) tissues was diminished by 270 administration, indicating that 270 could suppress the activated ER stress. Besides, we found LPS stimulation elevated the protein levels of LC3A and p62 in mice kidney and lung tissues, and the effect of LPS on these proteins in renal (Figure 8C,D) and lung tissues (Figure 8G,H) was normalized by 270 administration, suggesting that 270 could reverse the inhibition effect of LPS on autophagy.

\section{DISCUSSION AND CONCLUSIONS}

Although AKI and ALI are distinct entities, but they are frequently present simultaneously often inextricably linked in critically ill patients. Most intensive care unit patients will die from multiple rather than individual organ failure (Vincent, 2011). Sepsis is the leading cause of both AKI and ALI, and sepsis is characterized by systemic inflammation caused by infection (Yanet al. , 2019; Silveira et al. , 2021). LPS as a potent inducer of excessive inflammatory response, is widely used to establish AKI and ALI mouse models, which exhibit similar pathological changes to those found in human infectious sepsis (Ju et al. , 2018; Islam et al. , 2019). Currently, there are no effective strategies to prevent and cure AKI and ALI. Accumulating evidence suggests that anti-inflammation could be an effective therapeutic strategies for AKI and ALI treatment (Xie et al. , 2018; An et al. , 2019; Deng et al. , 2020). 
$\mathrm{NF}-x \mathrm{~B}$ is involved in the gene expression of LPS-induced inflammation response and the pathophysiology in septic AKI and ALI. Attenuated NF- $x \mathrm{~B}$ signaling has been shown previously to mediate protection against sepsis (Ibrahim et al. , 2020). Niu et al. showed that Harmine mitigates LPS-induced AKI through inhibition of the NF- $x$ B signaling pathway (Niu et al. , 2019). Chi et al. found that Limonene attenuated the pulmonary inflammatory responses induced by LPS in ALI via suppression of the NF- $x \mathrm{~B}$ and JNK pathways (Chi et al. , 2013). JNK is also an important mediator of inflammation that participated in the development of LPS-induced AKI and ALI. Additionally, Additionally, JNK can directly activate NF- $\chi \mathrm{B}$ by promoting I $\chi \mathrm{B}-\alpha$ degradation, and JNK and NF- $\varkappa \mathrm{B}$ can coordinate the inflammatory response. Grynberg et al. confirmed that administration of JNK inhibitors can protect against AKI (Grynberg et al. , 2017). Zheng et al. verified that JNK inhibitor SP600125 alleviated LPS-induced ALI (Zheng et al. , 2014). Thus, $\mathrm{NF}-\chi \mathrm{B}$ is an attractive therapeutic target for LPS-induced AKI and ALI. We designed and synthesized numerous NF- $x \mathrm{~B}$ inhibitors, and screen their transcriptional inhibition activity using an $\mathrm{NF}-x \mathrm{~B}$ reporter assay. We found compound 270 could inhibit NF-kB transcription with $\mathrm{IC}_{50}$ values with $2.49 \mu \mathrm{M}$. CETSA assay indicated that 270 directly interacts with NF- $x \mathrm{~B}$ proteins.

Macrophages are the principal immune cells mediator inflammation in kidney and lung tissues (Huang et al. , 2019). Thus, we examined the anti-inflammation activity of 270 in LPS-induced macrophages. We found that 270 inhibited the activation of NF- $x \mathrm{~B}$ and JNK signaling pathways and expression of pro-inflammatory genes in LPS-induced RAW 264.7 macrophages and BMDMs, and 270 suppressed the secretion of TNF- $\alpha$, IL-6 and MCP 1 in LPS-reduced BMDMs, suggesting that 270 possess the anti-inflammation activity in vitro.

We also verified that oral administration with 270 alleviated LPS-induced AKI and ALI in mice via improving inflammation in vivo. We discovered that 270 eliminated various pathological changes in renal and lung tissues, alleviated the enhanced of plasma Crea, BUN and pro-inflammation cytokines, and reversed the high expression of NGAL and KIM1 in kidney, accompanied by diminishing mRNA expression of proinflammation genes and blocking the activation of NF- $x \mathrm{~B}$ and JNK signaling pathways in kidney and lung tissues. Beside, MPO is a biomarker of neutrophils and macrophages accumulation manifests severity of inflammation in tissues (Islam et al. , 2019; Jiang et al. , 2019), and 270 attenuated the elevated MPO activity in renal and lung tissues, indicating that 270 possess the anti-inflammation activity in vivo.

Increasing evidences imply that pathogenesis of LPS-induced septic AKI and ALI are multifactorial and complex, involving the interplay among inflammation, oxidative stress, ER stress and autophagy (Ryter et al. , 2015; Zeng et al. , 2017; Yan et al. , 2018). It has been well documented that NF-xB signaling pathway activation was also closely related to the progression of oxidative stress, ER stress and autophagy (Muriach et al. , 2014; Huang et al. , 2016; Islam et al. , 2019). LPS accelerated lipid-peroxidation-mediated cytotoxic MDA formation, which is one maker of oxidative stress and can further contribute to organs injury (Baradaran Rahimi et al. , 2019; Islam et al. , 2019). We found MDA content was enhanced in LPS-induced mice kidney and lung tissues consistent with reported results, but mitigated by 270 .

LPS also can trigger ER stress, which becomes a major pathogenic factor in various diseases (Zeng et al. , 2017). Zhang et al. showed that MS-275 exhibits the nephroprotective effect on LPS-induced AKI by inhibiting ER stress (Zhang et al. , 2018a). Zeng et al. proved that 4-PBA ameliorates LPS-induced ALI through inactivating ER stress (Zeng et al. , 2017). In our study, we found BiP and CHOP, which were considered critical markers of ER stress, were elevated in LPS-induced mice kidney and lung tissues, and these alterations were restored by 270 treatment.

Studies have indicated that autophagy exerts a dual role in LPS-induced septic AKI and ALI (Zhang et al. , 2019). Mei et al. study showed that renal autophagy was rapidly induced by LPS, and inhibition of autophagy by chloroquine aggravated LPS-induced AKI in C57BL/6 mice (Meiet al. , 2016). But Zhao et al. pointed that dexmedetomidine protects against LPS-induced AKI by enhancing autophagy through inhibition of the PI3K/AKT/mTOR pathway (Zhao et al. , 2020). Zhang et al. found that the activation of autophagy in LPS-induced mice renal tissues, ACE2 activator RES could alleviate the severity of AKI via inhibiting autophagy and ACE2 inhibitor MLN-4760 aggravated the damage by promoting autophagy 
(Zhang et al. , 2019). However, Zhao et al. showed that LPS-induced ALI could be further exacerbated after suppression autophagy and enhancement of autophagy ameliorates ALI by weakening lung dysfunction (Zhao et al. , 2019). Both LC3A and p62 are usually used as biomarkers to monitor the level of autophagy, whose increased expression indicates the decreased autophagy (Song et al. , 2017). In our study, we discovered the inhibition of autophagy in LPS-induce mice kidney and lung tissues, as evidenced by the increment of LC3A and p62 protein levels, and pretreatment with 270 facilitated autophagy. The differences of these results in autophagy between we and others are likely associated with the use of various experimental models, animal background and the time-points monitored (Zhao et al. , 2020).

The development of LPS-induced AKI and ALI is not a mere singular mechanism, 270 alleviates LPS-induced septic AKI and ALI involving inflammation, oxidative stress, ER stress and autophagy, suggesting that 270 may be clinically viable. But several questions related to our findings remain to be answered. First, the detail mechanisms of 270 on oxidative stress, ER stress and autophagy need further study. Second, the cross-talk between inflammation, oxidative stress, ER stress and autophagy mediated by NF- $x \mathrm{~B}$ signaling pathway require further elucidation. In addition, we found ALI induced by intraperitoneal injection of LPS was less severe than AKI. Despite ALI could be induced by intranasal, intratracheal, intraperitoneal or intravenous administration of LPS, whereas LPS caused acute pulmonary damage in mice $24 \mathrm{~h}$ after the intranasal or intratracheal administration, intravenous and intraperitoneal administration did not lead to a tissue-specific or similar degree of lung injury (Chenet al. , 2010). To further verify the effects of 270 on ALI, the protective effects of 270 in ideal animal models of ALI should also be investigated.

In conclusion, our results reveal that NF- $\varkappa \mathrm{B}$ inhibitor 270 protects against LPS-induced AKI and ALI primarily through inhibiting NF- $\chi \mathrm{B}$ and JNK-mediated inflammation response, as well as improving oxidative stress, suppressing ER stress and promoting autophagy (Figure 9), and supply evidence that 270 may be a valuable therapeutic medicine against inflammation-related diseases.

\section{Author contributions}

YYY contributed to the hypothesis, study design, data analysis, experiments, and preparation of the paper; XQL, WPH, YNG, JJD, YTZ and SCC help performed the experiments, and all authors reviewed and approved the final version of the paper. All authors gave final approval to the publication.

\section{Declaration of transparency and scientific rigour}

This Declaration acknowledges that this paper adheres to the principles for transparent reporting and scientific rigour of preclinical research as stated in the BJP guidelines for Design \& Analysis, Immunoblotting and Immunochemistry, and Animal Experimentation, and as recommended by funding agencies, publishers and other organisations engaged with supporting research.

\section{Reference:}

An X, Sun X, Hou Y, Yang X, Chen H, Zhang P, et al. (2019). Protective effect of oxytocin on LPS-induced acute lung injury in mice. Scientific reports $\mathbf{9}(1): 2836$.

Badamjav R, Sonom D, Wu Y, Zhang Y, Kou J, Yu B, et al. (2020). The protective effects of Thalictrum minus L. on lipopolysaccharide-induced acute lung injury. Journal of ethnopharmacology 248: 112355.

Baradaran Rahimi V, Rakhshandeh H, Raucci F, Buono B, Shirazinia R, Samzadeh Kermani A, et al.(2019). Anti-Inflammatory and Anti-Oxidant Activity of Portulaca oleracea Extract on LPS-Induced Rat Lung Injury. Molecules 24 (1).

Chen H, Bai C, Wang X (2010). The value of the lipopolysaccharide-induced acute lung injury model in respiratory medicine. Expert review of respiratory medicine 4 (6): 773-783.

Chen Y, Guo S, Jiang K, Wang Y, Yang M, Guo M (2019). Glycitin alleviates lipopolysaccharide-induced acute lung injury via inhibiting NF-kappaB and MAPKs pathway activation in mice. International immunopharmacology 75: 105749. 
Chi G, Wei M, Xie X, Soromou LW, Liu F, Zhao S (2013). Suppression of MAPK and NF-kappaB pathways by limonene contributes to attenuation of lipopolysaccharide-induced inflammatory responses in acute lung injury. Inflammation36 (2): 501-511.

Deng JS, Jiang WP, Chen CC, Lee LY, Li PY, Huang WC, et al. (2020). Cordyceps cicadae Mycelia Ameliorate Cisplatin-Induced Acute Kidney Injury by Suppressing the TLR4/NF-kappaB/MAPK and Activating the HO-1/Nrf2 and Sirt-1/AMPK Pathways in Mice. Oxidative medicine and cellular longevity2020: 7912763.

Fu X, Schmitz FJ, Govindan M, Abbas SA, Hanson KM, Horton PA, et al. (1995). Enzyme inhibitors: new and known polybrominated phenols and diphenyl ethers from four Indo-Pacific Dysidea sponges. Journal of natural products58 (9): 1384-1391.

Grynberg K, Ma FY, Nikolic-Paterson DJ (2017). The JNK Signaling Pathway in Renal Fibrosis. Frontiers in physiology 8: 829.

Gumbert SD, Kork F, Jackson ML, Vanga N, Ghebremichael SJ, Wang CY, et al. (2020). Perioperative Acute Kidney Injury. Anesthesiology 132 (1): 180-204.

Huang WC, Lai CL, Liang YT, Hung HC, Liu HC, Liou CJ (2016). Phloretin attenuates LPS-induced acute lung injury in mice via modulation of the NF-kappaB and MAPK pathways. International immunopharmacology 40: 98-105.

Huang XT, Liu W, Zhou Y, Hao CX, Zhou Y, Zhang CY, et al. (2019). Dihydroartemisinin attenuates lipopolysaccharideinduced acute lung injury in mice by suppressing NFkappaB signaling in an Nrf2dependent manner. International journal of molecular medicine 44 (6): 2213-2222.

Ibrahim YF, Moussa RA, Bayoumi AMA, Ahmed AF (2020). Tocilizumab attenuates acute lung and kidney injuries and improves survival in a rat model of sepsis via down-regulation of NF-kappaB/JNK: a possible role of P-glycoprotein.Inflammopharmacology 28 (1): 215-230.

cute Kidney Injury in Mice via Regulating Inflammation and Oxidative Stress. Nutrients 11 (6).

Jiang Y, Quan J, Chen Y, Liao X, Dai Q, Lu R, et al. (2019). Fluorofenidone protects against acute kidney injury. FASEB journal : official publication of the Federation of American Societies for Experimental Biology33 (12): 14325-14336.

Ju M, Liu B, He H, Gu Z, Liu Y, Su Y, et al. (2018). MicroRNA-27a alleviates LPS-induced acute lung injury in mice via inhibiting in $\mathrm{fl}$ ammation and apoptosis through modulating TLR4/MyD88/NF-kappaB pathway. Cell cycle17 (16): 2001-2018.

Lee HS, Lee TH, Lee JH, Chae CS, Chung SC, Shin DS, et al. (2007). Inhibition of the pathogenicity of Magnaporthe grisea by bromophenols, isocitrate lyase inhibitors, from the red alga Odonthalia corymbifera. Journal of agricultural and food chemistry $\mathbf{5 5}$ (17): 6923-6928.

Martinez Molina D, Jafari R, Ignatushchenko M, Seki T, Larsson EA, Dan C, et al. (2013). Monitoring drug target engagement in cells and tissues using the cellular thermal shift assay. Science341 (6141): 84-87.

Mei S, Livingston M, Hao J, Li L, Mei C, Dong Z (2016). Autophagy is activated to protect against endotoxic acute kidney injury. Scientific reports 6: 22171.

Muriach M, Flores-Bellver M, Romero FJ, Barcia JM (2014). Diabetes and the brain: oxidative stress, inflammation, and autophagy. Oxidative medicine and cellular longevity 2014: 102158.

Nie Y, Wang Z, Chai G, Xiong Y, Li B, Zhang H, et al. (2019). Dehydrocostus Lactone Suppresses LPSinduced Acute Lung Injury and Macrophage Activation through NF-kappaB Signaling Pathway Mediated by p38 MAPK and Akt.Molecules 24 (8). 
Niu X, Yao Q, Li W, Zang L, Li W, Zhao J, et al. (2019). Harmine mitigates LPS-induced acute kidney injury through inhibition of the TLR4-NF-kappaB/NLRP3 inflammasome signalling pathway in mice. European journal of pharmacology849: 160-169.

Pak ES, Uddin MJ, Ha H (2020). Inhibition of Src Family Kinases Ameliorates LPS-Induced Acute Kidney Injury and Mitochondrial Dysfunction in Mice. International journal of molecular sciences 21 (21).

Ryter SW, Choi AM (2015). Autophagy in lung disease pathogenesis and therapeutics. Redox biology4: 215-225.

Silveira MAD, Capcha JMC, Sanches TR, de Sousa Moreira R, Garnica MS, Shimizu MH, et al. (2021). Green propolis extract attenuates acute kidney injury and lung injury in a rat model of sepsis. Scientific reports 11 (1):5925.

Song H, Yan C, Tian X, Zhu N, Li Y, Liu D, et al. (2017). CREG protects from myocardial ischemia/reperfusion injury by regulating myocardial autophagy and apoptosis. Biochimica et biophysica acta. Molecular basis of disease 1863 (8): 1893-1903.

Utkina NK, Denisenko VA, Scholokova OV, Virovaya MV, Gerasimenko AV, Popov DY, et al. (2001). Spongiadioxins A and B, two new polybrominated dibenzo-p-dioxins from an Australian marine sponge Dysidea dendyi. Journal of natural products 64 (2): 151-153.

Vincent JL (2011). Acute kidney injury, acute lung injury and septic shock: how does mortality compare? Contributions to nephrology 174: 71-77.

Wiemer DF, Idler DD, Fenical W (1991). Vidalols A and B, new anti-inflammatory bromophenols from the Caribbean marine red alga Vidalia obtusaloba. Experientia47 (8): 851-853.

Xie W, Lu Q, Wang K, Lu J, Gu X, Zhu D, et al. (2018). miR-34b-5p inhibition attenuates lung inflammation and apoptosis in an LPS-induced acute lung injury mouse model by targeting progranulin. Journal of cellular physiology 233 (9): 6615-6631.

Xu N, Fan X, Yan X, Li X, Niu R, Tseng CK (2003). Antibacterial bromophenols from the marine red alga Rhodomela confervoides. Phytochemistry 62 (8):1221-1224.

Xu X, Song F, Wang S, Li S, Xiao F, Zhao J, et al. (2004). Dibenzyl bromophenols with diverse dimerization patterns from the brown alga Leathesia nana. Journal of natural products 67 (10): 1661-1666.

Yan M, Shu S, Guo C, Tang C, Dong Z (2018). Endoplasmic reticulum stress in ischemic and nephrotoxic acute kidney injury. Annals of medicine 50 (5):381-390.

Yan XX, Zheng AD, Zhang ZE, Pan GC, Zhou W (2019). Protective effect of pantoprazole against sepsisinduced acute lung and kidney injury in rats. American journal of translational research 11 (8): 5197-5211.

Zeng M, Sang W, Chen S, Chen R, Zhang H, Xue F, et al. (2017). 4-PBA inhibits LPS-induced inflammation through regulating ER stress and autophagy in acute lung injury models. Toxicology letters 271: 26-37.

Zhang H, Zhang W, Jiao F, Li X, Zhang H, Wang L, et al. (2018a). The Nephroprotective Effect of MS-275 on Lipopolysaccharide (LPS)-Induced Acute Kidney Injury by Inhibiting Reactive Oxygen Species (ROS)Oxidative Stress and Endoplasmic Reticulum Stress. Medical science monitor : international medical journal of experimental and clinical research 24: 2620-2630.

Zhang W, Wang G, Zhou S (2018b). Protective Effects of Isoliquiritigenin on LPS-Induced Acute Lung Injury by Activating PPAR-gamma. Inflammation 41 (4):1290-1296.

Zhang X, Zheng J, Yan Y, Ruan Z, Su Y, Wang J, et al. (2019). Angiotensin-converting enzyme 2 regulates autophagy in acute lung injury through AMPK/mTOR signaling.Archives of biochemistry and biophysics 672: 108061 . 
Zhao JX, Yu YY, Wang SS, Huang SL, Shen Y, Gao XH, et al. (2018). Structural Elucidation and Bioinspired Total Syntheses of Ascorbylated Diterpenoid Hongkonoids A-D.Journal of the American Chemical Society 140 (7): 2485-2492.

Zhao X, Yu Z, Lv Z, Meng L, Xu J, Yuan S, et al. (2019). Activation of Alpha-7 Nicotinic Acetylcholine Receptors (alpha7nAchR) Promotes the Protective Autophagy in LPS-Induced Acute Lung Injury (ALI) In Vitro and In Vivo.Inflammation 42 (6): 2236-2245.

Zhao Y, Feng X, Li B, Sha J, Wang C, Yang T, et al. (2020). Dexmedetomidine Protects Against Lipopolysaccharide-Induced Acute Kidney Injury by Enhancing Autophagy Through Inhibition of the PI3K/AKT/mTOR Pathway. Frontiers in pharmacology 11: 128.

Zheng Y, Zhang M, Zhao Y, Chen J, Li B, Cai W (2014). JNK inhibitor SP600125 protects against lipopolysaccharide-induced acute lung injury via upregulation of claudin-4. Experimental and therapeutic medicine8 (1): 153-158.

Figure legends

Figure 1

Figure 1. Synthesis of bromophenol-chalcone derivatives

Figure 2

A<smiles>COc1ccc(C(=O)/C=C/c2cc(O)c(O)c(Br)c2)cc1O</smiles><smiles>COc1ccc(C(=O)/C=C/c2cc(O)c(O)c(Br)c2)cc1</smiles>

C

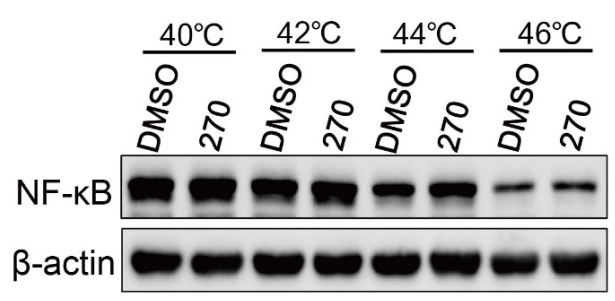

E

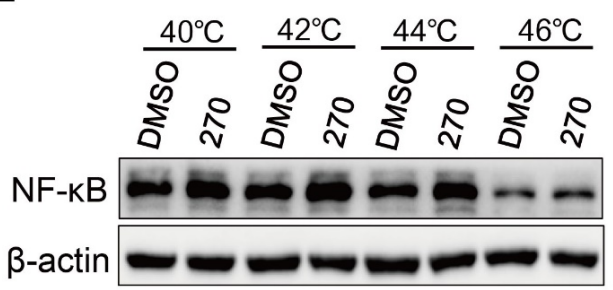

B

NF-kB transcriptional inhibition activity

\begin{tabular}{cc}
\hline Cmpd & $\mathrm{IC}_{50}(\mu \mathrm{M})$ \\
\hline 269 & $5.91 \pm 0.26$ \\
270 & $2.49 \pm 0.33$ \\
$\mathrm{PTL}$ & $1.05 \pm 0.23$ \\
\hline
\end{tabular}

D

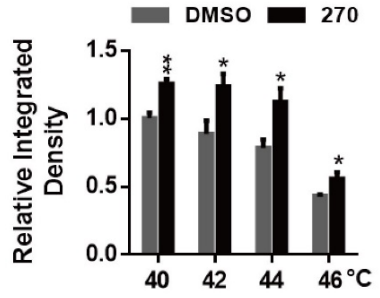

$\mathbf{F}$

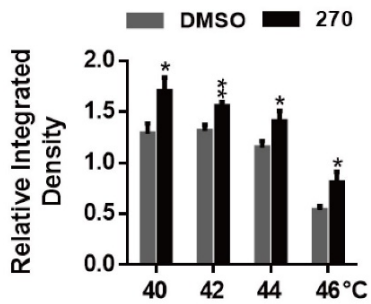




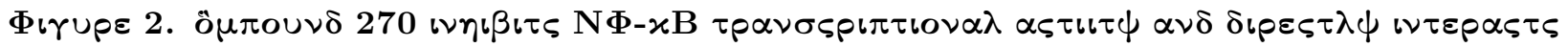
$\omega \iota \eta \eta \mathrm{N} \Phi-\varkappa \mathrm{B}$ เ HEK $293 \alpha \nu \delta$ PA $\Omega 264.7 \varsigma \varepsilon \lambda \lambda \varsigma$.

(A) Chemical structure of 269 and 270. (B) The NF- $x$ B transcriptional inhibition activity of 269 and 270. (C) Effect of 270 on NF- $x \mathrm{~B}$ protein stabilization in HEK 293 cells. HEK 293 cells transfected with NF- $x \mathrm{~B}$ expression plasmid were treated with $5 \mu \mathrm{M} 270$ for $3 \mathrm{~h}$ before heated at different temperatures. Protein levels were analyzed by western blotting. (D) Protein band densities shown in (C) were quantified by normalizing to $\beta$-actin. (E) Effect of 270 on NF- $x$ B protein stabilization in RAW 264.7 macrophages. RAW 264.7 macrophages were treated with $5 \mu \mathrm{M} 270$ for $3 \mathrm{~h}$ before heated at different temperatures. Protein levels were analyzed by western blotting. (F) Protein band densities shown in (E) were quantified by normalizing to $\beta$-actin. Data are mean $\pm \mathrm{SEM}, \mathrm{n}=5 .{ }^{*} \mathrm{P}<0.05,{ }^{*} \mathrm{P}<0.01,{ }^{* * *} \mathrm{P}<0.001$ versus DMSO.

Figure 3

A

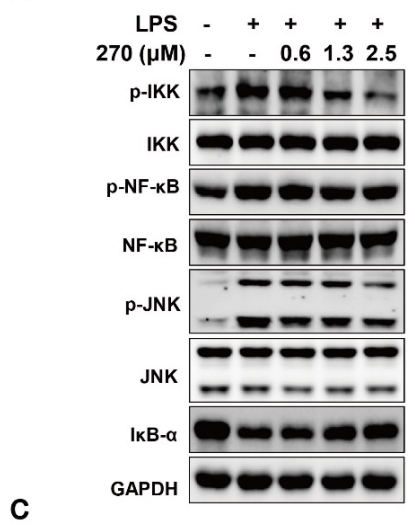

B
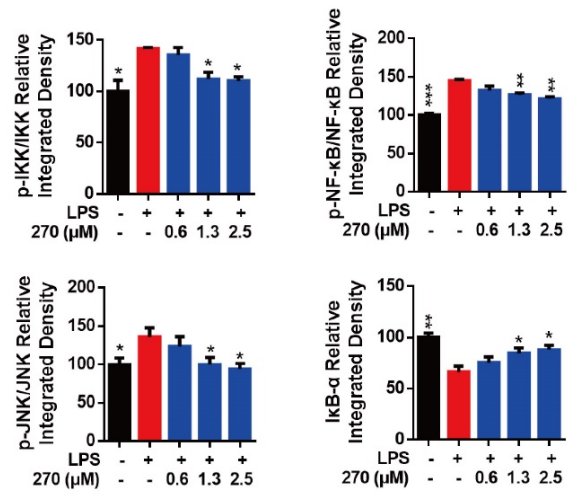
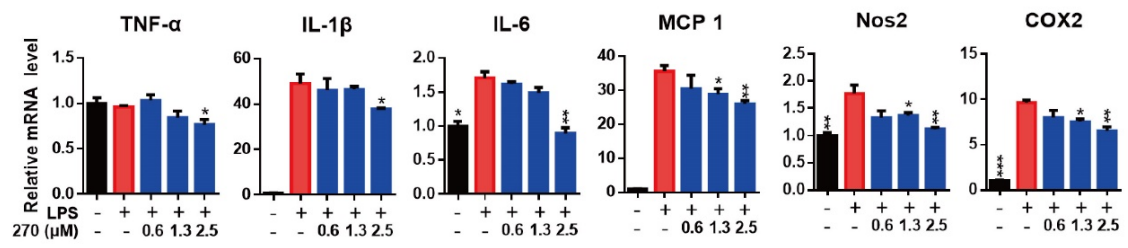

Figure 3. Compound 270 suppresses the LPS-induced inflammatory response in RAW 264.7 macrophages.

(A) RAW 264.7 cells were pre-incubated with 270 for $4 \mathrm{~h}$ and then treated with LPS $(100 \mathrm{ng} / \mathrm{ml})$ for 30 min. Total cell lysates were subjected to western blot. (B) The protein levels shown in (A) were quantified by densitometry analysis. (C) RAW 264.7 cells were treated with LPS (100 ng/ml) alone or together with 270 for $24 \mathrm{~h}$. Relative mRNA level of each pro-inflammatory gene was analyzed by use of RT-PCR and normalized by GAPDH. The data are presented as the means \pm SEM, $\mathrm{n}=5 .{ }^{*} \mathrm{P}<0.05,{ }^{* *} \mathrm{P}<0.01,{ }^{* * *} \mathrm{P}<$ 0.001 versus LPS group.

Figure 4 
A

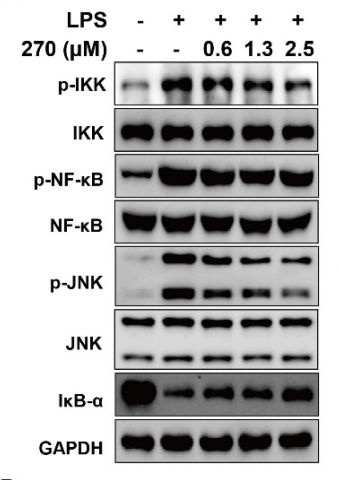

D

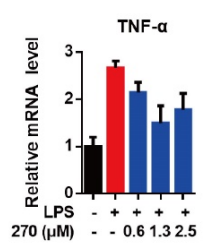

B

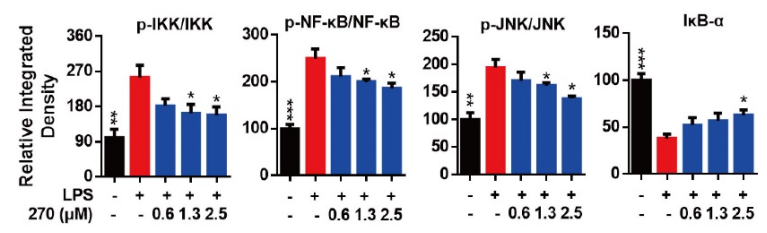

C
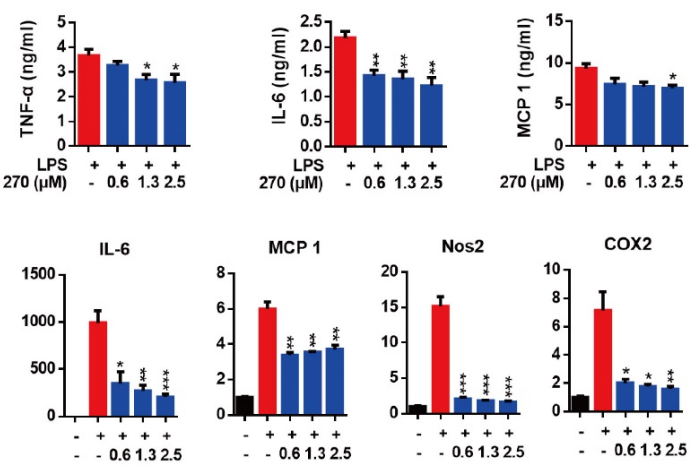

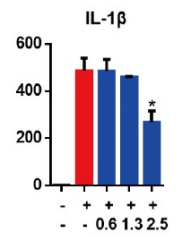

Figure 4. Compound 270 ameliorates the LPS-induced inflammatory response in bone marrow derived macrophages (BMDMs)

(A) BMDMs were pretreated with 270 for $4 \mathrm{~h}$ before LPS $(100 \mathrm{ng} / \mathrm{mL})$ treatment for $30 \mathrm{~min}$. Protein was isolated from the BMDMs and inflammation signaling proteins were assessed by western blot. (B) The protein levels shown in (A) were quantified by densitometry analysis. (C) BMDMs were treated with LPS (100 ng/ml) alone or together with 270 for $24 \mathrm{~h}$. The concentration of TNF- $\alpha$, IL-1 $\beta$ and MCP 1 in the medium were examined by ELISA. (D) BMDMs were treated with LPS (100 ng/ml) alone or together with 270 for $24 \mathrm{~h}$. Total RNA was extracted from BMDMs and the relative mRNA level of each pro-inflammatory gene was detected by use of RT-PCR and normalized by GAPDH. The data are presented as the means \pm SEM, $\mathrm{n}=5 .{ }^{*} \mathrm{P}<0.05,{ }^{* *} \mathrm{P}<0.01,{ }^{* * *} \mathrm{P}<0.001$ versus LPS group.

Figure 5

A

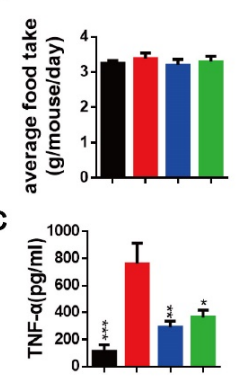

B
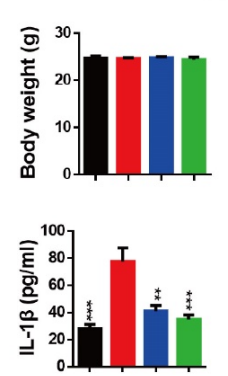
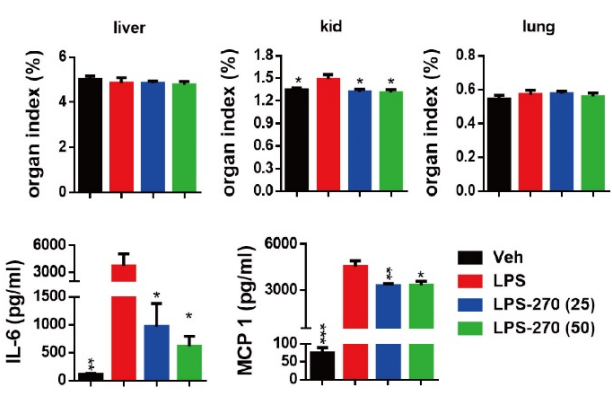

Figure 5. Compound 270 alleviates the excessive biosynthesis of inflammatory cytokines induced by intraperitoneal injection of LPS in C57BL/6J mice

C57BL/6J mice, at the age of 8 weeks, randomly divided into four groups, and the mice in LPS-270-(25) and LPS-270-(50) were administered $25 \mathrm{mg} / \mathrm{kg}$ and $50 \mathrm{mg} / \mathrm{kg} 270$ via oral gavage for 14 days. At the end of day 18 , the mice were treated by intraperitoneal injection with LPS $(10 \mathrm{mg} / \mathrm{kg})$, and $24 \mathrm{~h}$ after LPS injection, 
the animals were killed. (A)The effects of 270 on food take and body weight. (B) The effects of 270 on the liver, kidney and lung index. (C) The concentration of cytokines in the plasma was detected by ELISA. The data are presented as the means $\pm \mathrm{SEM}, \mathrm{n}=6 .{ }^{*} \mathrm{P}<0.05,{ }^{* *} \mathrm{P}<0.01,{ }^{* * *} \mathrm{P}<0.001$ compared with the LPS group.

Figure 6

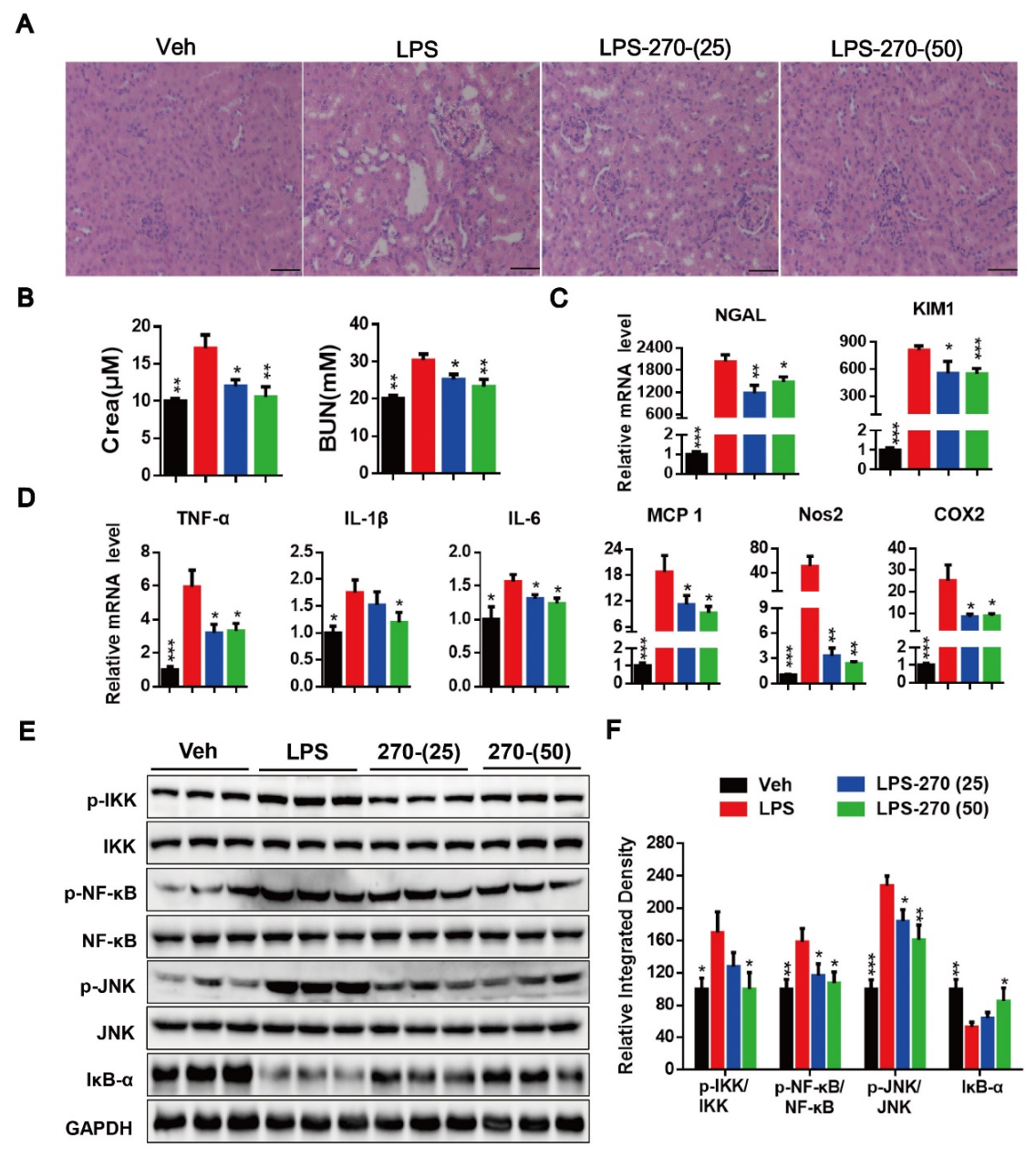

Figure 6. Compound 270 improves the mice acute kidney injury caused by intraperitoneal injection of LPS through attenuating renal inflammation state

(A) HE staining of kidney sections after $24 \mathrm{~h}$ LPS-induced kidney injury. Scale bar $=200 \mu \mathrm{m}$. (B) The concentrations of plasma Crea and BUN. (C) The relative mRNA level of NGAL and KIM1 in mice kidney tissue and normalized by GAPDH. (D) The relative mRNA level of each pro-inflammatory gene in renal tissues was detected by use of RT-PCR and normalized by GAPDH. (E) Western blot analysis of inflammatory signaling protein expression in kidney tissues. (F) Relative protein expression levels were determined by densitometric quantification of the immunoblots shown in (E). The data are presented as the means \pm SEM, $\mathrm{n}=6 .{ }^{*} \mathrm{P}<0.05,{ }^{*} \mathrm{P}<0.01,{ }^{* * *} \mathrm{P}<0.001$ compared with the LPS group.

Figure 7 
A

Aeh

LPS

LPS-270-(25)

LPS-270-(50)
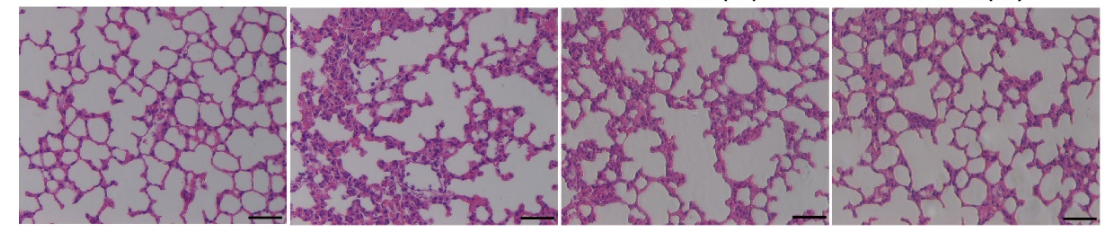

B

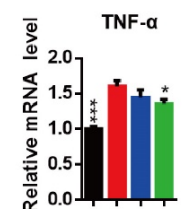

$\left.\begin{array}{l}2.5 \\ 2.0 \\ 1.5 \\ 1.0 \\ 0.5 \\ 0.0\end{array}\right]$

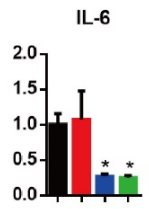

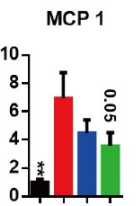

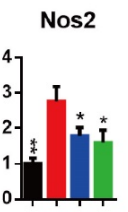

$\operatorname{cox} 2$

C

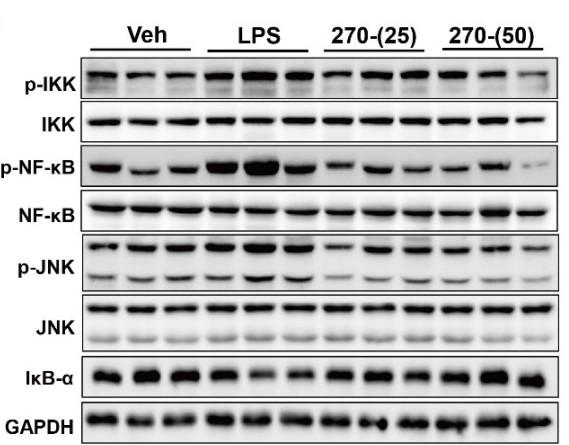

D

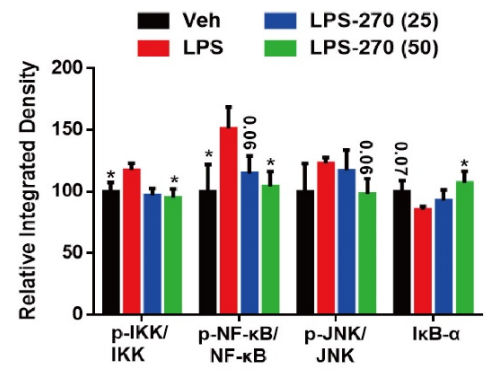

Figure 7. Compound 270 relieves the mice acute lung injury triggered by intraperitoneal injection of LPS through eliminating lung inflammation state

(A) Histological assessment of lung tissues was evaluated by HE staining. Scale bar $=200 \mu \mathrm{m}$. (B) The relative mRNA levels of pro-inflammation genes in lung tissues were quantified by RT-PCR normalized by GAPDH. (C) The protein expression of NF- $x \mathrm{~B}$ and JNK signaling pathways in lung tissues was measured by western blot analysis. (D) Quantification of the relative protein expression shown in (C) was performed by densitometric analysis. The data are presented as the means \pm SEM, $\mathrm{n}=6 .{ }^{*} \mathrm{P}<0.05,{ }^{* *} \mathrm{P}<0.01,{ }^{* * *} \mathrm{P}$ $<0.001$ compared with the LPS group.

Figure 8 
A

B

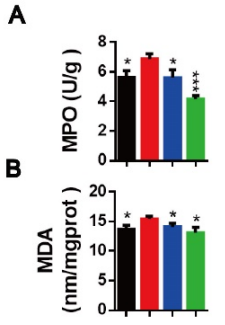

E
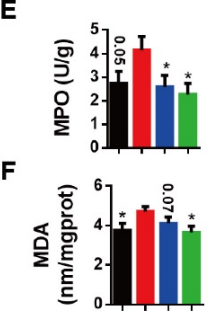

C

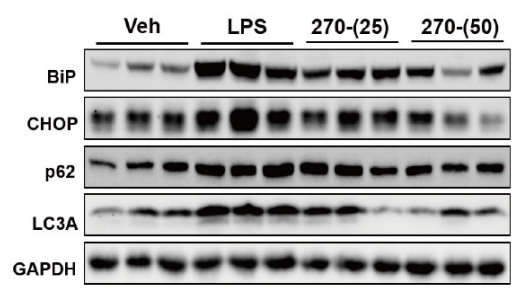

G

Veh LPS 270-(25) 270-(50)

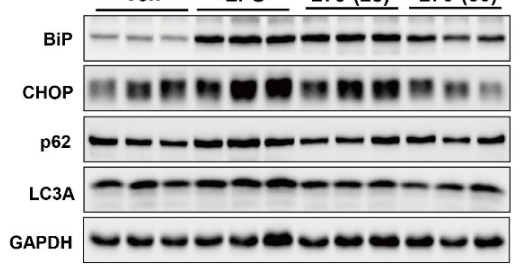

D

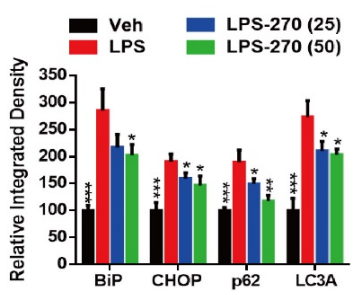

$\mathrm{H}$

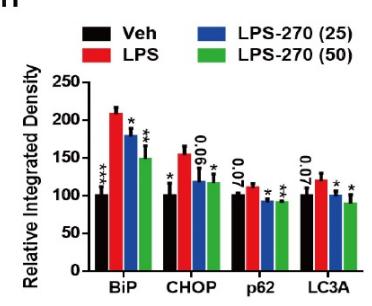

Figure 8. Compound 270 affects various molecular mechanism involved in the pathogenesis of acute kidney and lung injury challenged with intraperitoneal injection of LPS

(A) The activity of MPO in kidney tissues. (B) The content of MDA in kidney tissues. (C) The ER stress relative proteins $\mathrm{BiP}$ and $\mathrm{CHOP}$, as well as the autophagy associated proteins LC3A and p62 in kidney tissues were determined by western blotting. (D) The protein levels shown in (C) were quantified by densitometry analysis. (E) The activity of MPO in lung tissues. (F) The content of MDA in lung tissues. (G) The ER stress relative proteins BiP and CHOP, as well as the autophagy associated proteins LC3A and p62 in lung tissues were determined by western blotting. $(\mathrm{H})$ The protein levels shown in $(\mathrm{G})$ were quantified by densitometry analysis. The data are presented as the means $\pm \mathrm{SEM}, \mathrm{n}=6$. ${ }^{*} \mathrm{P}<0.05,{ }^{* *} \mathrm{P}<0.01,{ }^{* * *} \mathrm{P}<$ 0.001 compared with the LPS group.

Figure 9 


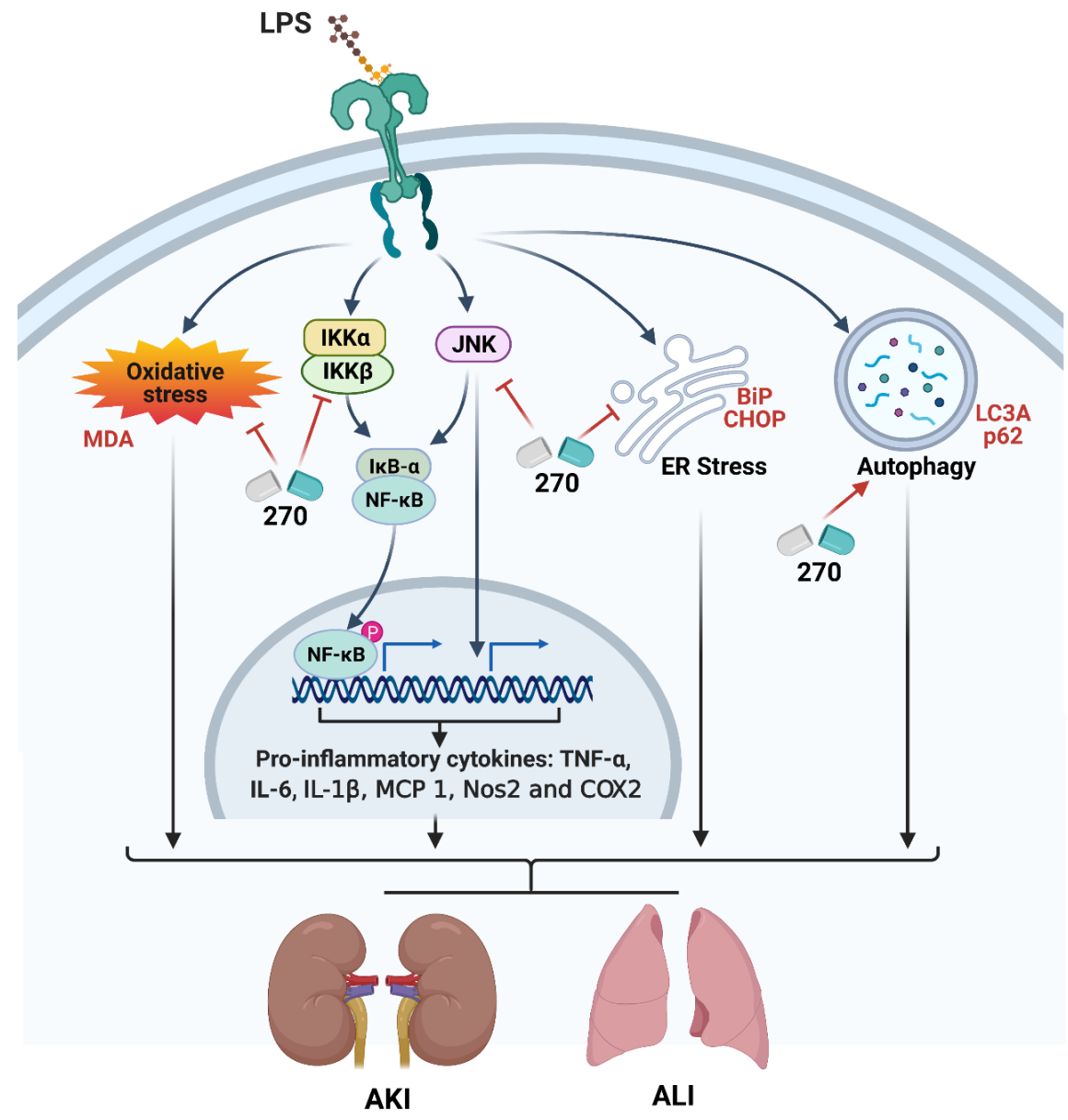

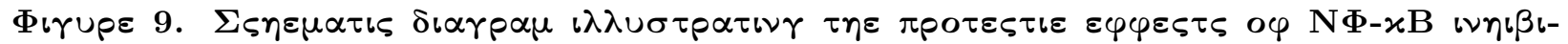

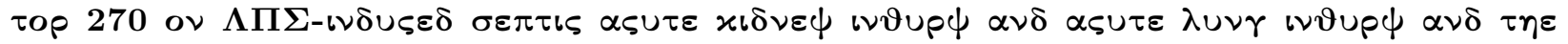

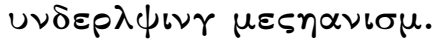

LPS can induce NF- $x \mathrm{~B}$ activation via IKK and JNK pathways, and then translocates from the cytoplasm to nucleus, which triggers the transcription of target genes. LPS can trigger oxidative stress evidencing by the increase of MDA. LPS can activate ER stress via up-regulating BiP and CHOP protein level. LPS can inhibit autophagy via accumulating LC3A and p62 protein. Oral treatment with 270 protected LPS induced AKI and ALI by suppressing IKK/ NF- $\varkappa$ B and JNK signaling pathway improving inflammation, inhibiting oxidative stress and ER stress, and activating autophagy. 